J3eA, Journal sur l'enseignement des sciences et technologies de l'information et des systèmes, Volume 4, Hors-Série 1, 2 (2005)

DOI : http://dx.doi.org/10.1051/bib-j3ea:2005602

(C) EDP Sciences, 2005

Contribution à l'étude de la stabilité des systèmes distribués Application aux réseaux de bord d'avions

S. Pierfederici ${ }^{1}$, R. Meuret ${ }^{2}$, F. Meibody-Tabar ${ }^{1}$ et B. Davat ${ }^{1}$

${ }^{1}$ GREEN-INPL, CNRS UMR 7037

${ }^{2}$ HISPANO-SUIZA, Groupe SNCEMA

serge.pierfederici@ensem.inpl-nancy.fr 


\title{
Contribution à l'étude de la stabilité des systèmes distribués - Application aux réseaux de bord d'avions
}

\author{
S. Pierfederici*, R. Meuret**, F. Meibody-Tabar*, B. Davat* \\ *GREEN-INPL, CNRS UMR 7037 \\ **HISPANO-SUIZA, Groupe SNCEMA \\ email : serge.pierfederici@ensem.inpl-nancy.fr
}

\begin{abstract}
Résumé : Dans cet article, nous présentons deux approches permettant d'étudier la stabilité d'un ensemble de dispositifs électriques interconnectés. La première établit des contraintes exprimées sous forme d'impédance entre les divers dispositifs. Un exemple d'application traitant le cas d'un ensemble onduleur-machine synchrone muni de son filtre d'entrée est présenté. La seconde approche s'applique au cas des systèmes électriques munis d'une commande interne. Il est alors possible par la commande d'adapter les dynamiques de la source d'énergie à celle de ses charges. Le cas d'un redresseur commandé alimentant $\mathrm{N}$ ensembles convertisseurs-machine synchrone est ensuite présenté.
\end{abstract}

\section{INTRODUCTION}

La multiplication des charges connectées sur le même bus continu engendre des problèmes d'interaction électrique entre les convertisseurs [1-2]. Il devient impératif d'analyser et d'optimiser ces réseaux et les interactions entre les divers sous systèmes [3-4]. Dans cet article, nous présentons les méthodes permettant d'étudier la stabilité du bus continu d'un système distribué constitué d'une source électrique alimentant un bus continu lui-même connecté à un ensemble de charges électriques. Deux approches sont développées. La première expose les procédés qui permettent de prouver la stabilité d'un système distribué sans modification des commandes de chaque élément [5-6]. Différents critères sont exposés, notamment les critères de Middelbrook, et d'ESAC. Un exemple d'application permet d'étudier l'interaction entre un onduleur alimentant une machine synchrone à aimant permanent et son filtre d'entrée connecté à un réseau d'avion. L'influence des modes de régulations utilisés pour le contrôle moteur sur le dimensionnement du filtre d'entrée est traité. Une méthode basée sur la compensation des variations d'énergie stockée dans le condensateur de filtrage permet une diminution significative des ondulations de tension de l'étage continu lors de fortes variations de puissance. La seconde approche utilise une structure de découplage généralisée qui permet un parfait contrôle du bus continu lorsqu'on dispose d'organe de commande coté source continue. Un exemple est traité mettant en œuvre un redresseur commandé alimentant un ensembles onduleur/machine synchrone à aimant. Dans un premier temps, tous les régulateurs utilisés pour le contrôle des moteurs sont supposés linéaires (contrôleur de type P.I). Cette hypothèse permet après linéarisation autour d'un point de fonctionnement, d'exhiber une structure de découplage du système. On montre que grâce à elle, il est possible de diminuer fortement la taille de la capacité de stockage d'énergie du bus continu. De plus, le découplage proposé ne change en rien la commande des ensembles convertisseurs-machines. Seule la commande du redresseur doit être modifiée pour assurer le contrôle de la tension continue, ce qui permet d'étendre la structure de contrôle proposée à tous les types de contrôle moteur. Des résultats de simulation avec des contrôleurs de courants de type RST et glissant sont présentés pour valider l'approche proposée.

II. APPROCHE FREQUENCIELLE POUR L'ETUDE DE LA STABILITE DES SYSTEMES DISTRIBUEES.

\section{A. Cas de 2 dispositifs connectés en parallèle}

Pour étudier la stabilité d'un ensemble de dispositifs électriques inter-connectés, on fait l'hypothèse que pris indépendamment chaque élément est stable. On appelle « dispositif » tout appareillage électrotechnique passif (ensemble redresseur avec son filtre d'entrée par exemple) ou actif (redresseur commandé par exemple). On suppose connu l'impédance d'entrée et de sortie de chaque dispositif. Un schéma de principe correspondant à la mise en cascade de deux dispositifs électriques est donné sur la figure 1.

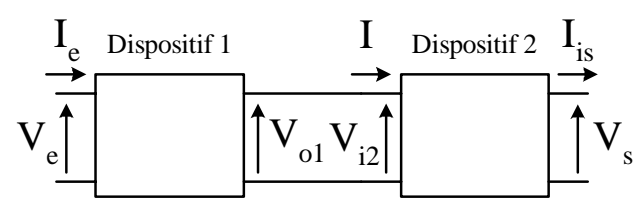

Figure 1. Mise en cascade de deux dispositifs électriques

La stabilité de chaque dispositif étant assurée au point de fonctionnement, il est alors possible, après 
linéarisation autour du point d'équilibre et transformation de Laplace, de modéliser les deux dispositifs électriques sous une forme quadripôle :

$$
\left|\begin{array}{c}
\tilde{\mathrm{v}}_{01} \\
\tilde{\mathrm{i}}_{\mathrm{e}}
\end{array}\right|=\left|\begin{array}{cc}
\mathrm{T}_{\mathrm{v} 1} & \mathrm{Z}_{\text {out }} \\
\frac{1}{\mathrm{Z}_{\mathrm{e} 1}} & -\mathrm{T}_{\mathrm{c} 1}
\end{array}\right| \cdot\left|\begin{array}{c}
\tilde{\mathrm{v}}_{\mathrm{e}} \\
\tilde{\mathrm{i}}
\end{array}\right| \quad\left|\begin{array}{c}
\tilde{\mathrm{v}}_{02} \\
\tilde{\mathrm{i}}
\end{array}\right|=\left|\begin{array}{cc}
\mathrm{T}_{\mathrm{v} 2} & \mathrm{Z}_{\mathrm{s} 2} \\
\frac{1}{\mathrm{Z}_{\text {in }}} & -\mathrm{T}_{\mathrm{c} 2}
\end{array}\right| \cdot\left|\begin{array}{c}
\tilde{\mathrm{v}}_{01} \\
-\tilde{\mathrm{i}_{\mathrm{s}}}
\end{array}\right|
$$

où $\mathrm{T}_{\mathrm{v} 1}$ et $\mathrm{T}_{\mathrm{V} 2}$ représentent les fonctions de transfert en tension, $\mathrm{T}_{\mathrm{c} 1}$ et $\mathrm{T}_{\mathrm{c} 2}$ les fonctions de transfert en courant. $\tilde{\mathrm{X}}$ représente les variations de la variable $\mathrm{X}$ autour du point de fonctionnement. La mise en cascade des deux dispositifs est possible si la fonction de transfert $\hat{\mathrm{v}}_{02} / \hat{\mathrm{v}}_{\mathrm{e}}$ est stable. Supposons que le courant de charge $I_{s}$ reste constant $\left(\tilde{i}_{s}=0\right)$, il vient alors :

$$
\begin{gathered}
\tilde{\mathrm{v}}_{01}=\mathrm{T}_{\mathrm{v} 1} \cdot \tilde{\mathrm{v} e}-\mathrm{Z}_{\text {out }} \cdot \tilde{\mathrm{i}}=\mathrm{T}_{\mathrm{v} 1} \cdot \tilde{\mathrm{v} e}-\mathrm{Z}_{\mathrm{s} 1} \cdot \frac{\tilde{\mathrm{v}}_{01}}{\mathrm{Z}_{\text {in }}} \\
\text { d'où }: \hat{\mathrm{v}}_{01}=\frac{\mathrm{T}_{\mathrm{v} 1} \cdot \hat{\mathrm{v}}_{\mathrm{e}}}{1+\frac{\mathrm{Z}_{\text {out }}}{\mathrm{Z}_{\text {in }}}} \text { et }: \frac{\hat{\mathrm{v}}_{02}}{\hat{\mathrm{v}}_{\mathrm{e}}}=\frac{\mathrm{T}_{\mathrm{v} 1} \cdot \mathrm{T}_{\mathrm{v} 2}}{1+\frac{\mathrm{Z}_{\text {out }}}{\mathrm{Z}_{\text {in }}}}
\end{gathered}
$$

On pose alors $T_{m}=Z_{\text {out }} / Z_{\text {in }}$. Puisque $T_{v 1}$ et $T_{v 2}$ sont des fonctions de transfert stables, une condition suffisante de stabilité est que la fonction de transfert $\mathrm{T}_{\mathrm{m}}$ soit stable. L'application du critère de Nyquist permet d'étudier la stabilité de cette association. Basées sur l'étude de ce tracé, différentes méthodes ont été proposées dans la littérature (critère de Middelbrook, critère d'ESAC, methode MGMP [1, 2, $5,6])$.

\section{B. Cas de n dispositifs connectés en parallèle}

Comme dans le cas précédant, la stabilité de la fonction de transfert de la tension pour chaque convertisseur aboutit à :

$\frac{\hat{\mathrm{v}}_{0 \mathrm{j}}}{\hat{\mathrm{v}}_{\mathrm{e}}}=\frac{\mathrm{T}_{\mathrm{v} 1} \cdot \mathrm{T}_{\mathrm{vj}}}{1+\mathrm{Z}_{\text {out }} \cdot \mathrm{T}_{\mathrm{m}}}$

avec : $\mathrm{T}_{\mathrm{m}}=\mathrm{Z}_{\text {out }} \cdot \sum_{\mathrm{j}=1}^{\mathrm{n}} \frac{1}{\mathrm{Z}_{\mathrm{in}_{\mathrm{j}}}}$ et $: \mathrm{T}_{\mathrm{j}}=\frac{\mathrm{Z}_{\text {out }}}{\mathrm{Z}_{\mathrm{in}_{\mathrm{j}}}}$

Pour prouver la stabilité du système distribué, les relations suivantes doivent être vérifiées $\forall \mathrm{j} \in\{1, \ldots, \mathrm{N}\}$

$$
\begin{aligned}
& \left|Z_{\text {in } \mathrm{j}}\right|_{\mathrm{dB}}-\left|Z_{\text {out }}\right|_{\mathrm{dB}}>\mathrm{MG}+20 \cdot \log \left(\frac{\mathrm{P}_{\mathrm{j}}}{\mathrm{P}_{\mathrm{s}}}\right) \\
& \text { real } \cdot\left(\left(\frac{\mathrm{Z}_{\text {out }}}{\mathrm{Z}_{\text {in } \mathrm{j}}}\right)\right) \geq-\operatorname{Arccos}(\mathrm{PM}) \cdot\left(\frac{\mathrm{P}_{\mathrm{j}}}{\mathrm{P}_{\mathrm{s}}}\right)
\end{aligned}
$$

où $\mathrm{P}_{\mathrm{j}}$ représente la puissance fournie par chaque dispositif à leur charge respective et $\mathrm{P}_{\mathrm{s}}$ représente la puissance totale fournie par la source. La figure 2 représente un exemple de tracé satisfaisant ces deux relations.

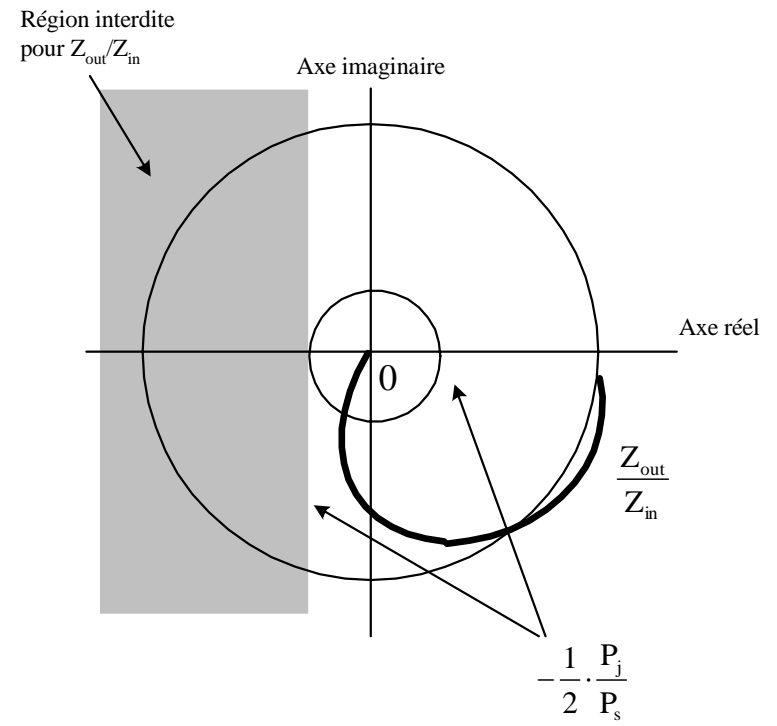

Figure 2 : Spécification individuelle de charge avec une marge de gain MG de $6 \mathrm{db}$ et une marge de phase PM de $60^{\circ}$

\section{APPLICATION A UN ENSEMBLE REDRESSEUR FILTRE ET ONDULEUR MACHINE SYNCHRONE A AIMANT}

\section{A. Présentation du système}

Dans cette partie, on étudie la stabilité d'un système constitué d'un ensemble auto transformateur redresseurs (figure 3) alimentant un ensemble onduleur machine synchrone à aimant régulée en vitesse. La mise en parallèle des deux ponts redresseurs se fait par l'intermédiaire de deux inductances d'interphase présentant une inductance de fuite L supposée connue. Pour ce faire, l'étude est scindée en deux parties. L'une permet d'obtenir l'impédance d'entrée de l'ensemble onduleur-machine, l'autre l'impédance de sortie de la source d'énergie.

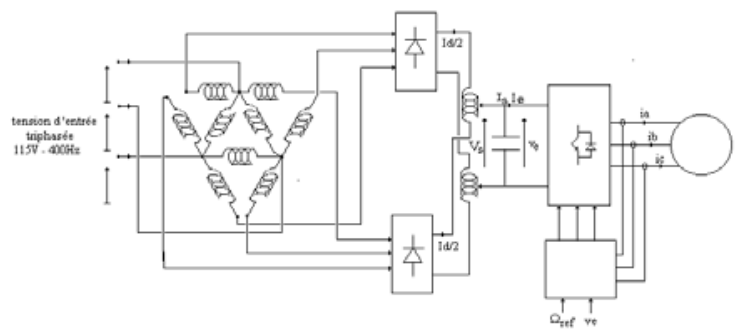

Figure 3 : Schéma de principe du dispositif expérimental

B. Calcul de l'impédance d'entrée de l'ensemble onduleur-machine synchrone

On se place autour d'un point de fonctionnement et on considère de petites variations autour du point d'équilibre. En supposant que la constante de temps mécanique est beaucoup plus grande que la plus grande des constantes de temps électrique, on néglige ainsi les variations de vitesse dans la modélisation. L'impédance d'entrée est alors donnée par l'expression $\mathrm{Z}_{\mathrm{e}}=\tilde{\mathrm{v}}_{\mathrm{e}} / \tilde{\mathrm{i}}_{\mathrm{e}}=\mathrm{Z}_{\text {in }}$ où $\tilde{\mathrm{v}}_{\mathrm{e}}$ et $\tilde{\mathrm{i}}_{\mathrm{e}}$ représentent les variations de la tension et du courant d'entrée autour du point de fonctionnement (figure 4). 


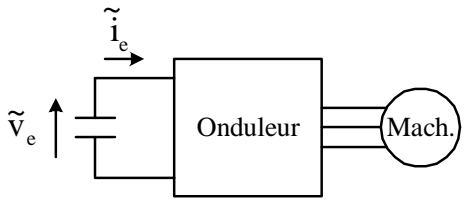

Figure 4. Schéma de principe de l'ensemble convertisseur moteur

En supposant les pertes par commutation nulles dans l'onduleur, la loi de conservation de puissance s'écrit :

$\mathrm{v}_{\mathrm{e}} \cdot \mathrm{i}_{\mathrm{e}}=\mathrm{v}_{\mathrm{d}} \cdot \mathrm{i}_{\mathrm{d}}+\mathrm{v}_{\mathrm{q}} \cdot \mathrm{i}_{\mathrm{q}}$

avec : $\left[\begin{array}{c}\mathrm{v}_{\mathrm{d}} \\ \mathrm{v}_{\mathrm{q}}\end{array}\right]=\mathrm{G} \cdot\left[\begin{array}{c}\mathrm{v}_{\mathrm{d}}{ }^{*} \\ \mathrm{v}_{\mathrm{q}}{ }^{*}\end{array}\right] \quad$ où : $\mathrm{G}=\frac{\mathrm{v}_{\mathrm{e}}}{2 \cdot \mathrm{p}_{\mathrm{m}}}$ est le gain de l'onduleur, $\mathrm{p}_{\mathrm{m}}$ l'amplitude de la porteuse et $\mathrm{V}_{\mathrm{d}}{ }^{*}$ et $\mathrm{V}_{\mathrm{q}}{ }^{*}$ les tensions de commande d'axes $\mathrm{d}$ et $\mathrm{q}$ de l'onduleur. Pour de petites variations autour du point de fonctionnement, le modèle linéarisé conduit à :

$$
\begin{aligned}
& \tilde{v}_{e} \cdot I_{e}+V_{e} \cdot \tilde{i}_{e}=\tilde{v}_{d} \cdot I_{d}+V_{d} \cdot \tilde{i}_{d}+\tilde{v}_{q} \cdot I_{q}+V_{q} \cdot \tilde{i}_{q} \\
& {\left[\begin{array}{l}
\tilde{v}_{d} \\
\tilde{v}_{q}
\end{array}\right]=\frac{\tilde{v}_{e}}{2 \cdot p_{m}} \cdot\left[\begin{array}{c}
v_{d}^{*} \\
v_{q}^{*}
\end{array}\right]+\frac{V_{e}}{2 \cdot p_{m}} \cdot\left[\begin{array}{c}
\tilde{v}_{d} \\
\tilde{v}_{q}
\end{array}\right]}
\end{aligned}
$$

Le principe de commande utilisé est présenté sur la figure 5. Un terme additionnel apparaît dans la structure de contrôle. A la référence de courant d'axe q classique est additionné un terme proportionnel aux variations de la tension d'entrée autour de sa valeur moyenne. En fait, on demande au moteur d'adapter sa demande de puissance afin de lutter contre les fluctuations de la tension d'entrée, notamment lors du démarrage de la machine.

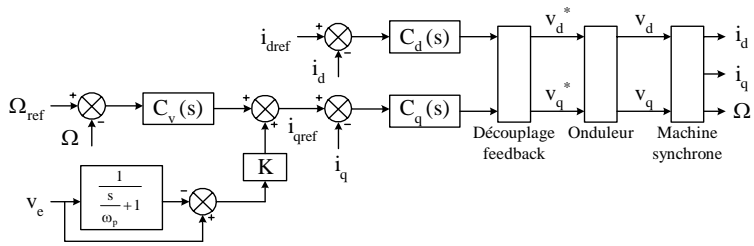

Figure 5. Schéma de contrôle de la machine synchrone à aimant permanent

En utilisant les équations standards de la machine synchrone à aimant permanent et le schéma de contrôle proposé sur la figure 6, il vient alors :

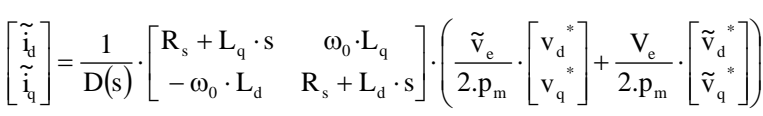

$$
\begin{aligned}
\text { où : } \mathrm{D}(\mathrm{s})=\mathrm{R}_{\mathrm{s}}{ }^{2} \cdot\left[\left(1+\tau_{\mathrm{d}} \cdot \mathrm{s}\right) \cdot\left(1+\tau_{\mathrm{q}} \cdot \mathrm{s}\right)+\omega_{0}{ }^{2} \cdot \tau_{\mathrm{d}} \cdot \tau_{\mathrm{q}}\right] \\
\tau_{\mathrm{d}}=\frac{\mathrm{L}_{\mathrm{d}}}{\mathrm{R}_{\mathrm{s}}} \text { et }: \tau_{\mathrm{q}}=\frac{\mathrm{L}_{\mathrm{q}}}{\mathrm{R}_{\mathrm{s}}}
\end{aligned}
$$

En combinant les relations (1) et (2), il est alors possible d'exprimer les variations des grandeurs d'entrée en fonction des variations des courants d'axe d et $\mathrm{q}$ de la machine :

$\tilde{v}_{e} \cdot I_{e}+V_{e} \cdot \tilde{i}_{e}=C(s) \cdot \tilde{i}_{d}+D(s) \cdot \tilde{i}_{4}$

avec :

$$
\begin{aligned}
& \mathrm{C}(\mathrm{s})=\mathrm{V}_{\mathrm{d}}+\mathrm{I}_{\mathrm{d}} \cdot\left(\mathrm{R}_{\mathrm{s}}+\mathrm{L}_{\mathrm{d}} \cdot \mathrm{s}\right)+\omega_{0} \cdot \mathrm{L}_{\mathrm{d}} \cdot \mathrm{I}_{\mathrm{q}} \\
& \mathrm{D}(\mathrm{s})=\mathrm{V}_{\mathrm{q}}+\mathrm{I}_{\mathrm{q}} \cdot\left(\mathrm{R}_{\mathrm{s}}+\mathrm{L}_{\mathrm{q}} \cdot \mathrm{s}\right)-\omega_{0} \cdot \mathrm{L}_{\mathrm{q}} \cdot \mathrm{I}_{\mathrm{d}}
\end{aligned}
$$

A ce stade, il est nécessaire d'introduire la méthode de découplage choisie pour la régulation des courants d'axes d et q. On va détailler les calculs dans le cas d'un découplage de type Feedforward. Les tensions de commande d'axes d, q vérifient alors :

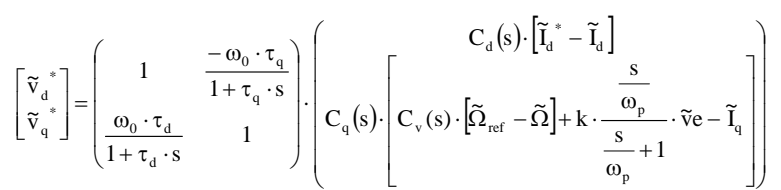

où $\mathrm{C}_{\mathrm{d}}(\mathrm{s})$ et $\mathrm{C}_{\mathrm{q}}(\mathrm{s})$ représentent les correcteurs de courant d'axes d et q et $\mathrm{C}_{\mathrm{v}}(\mathrm{s})$ le correcteur de vitesse.

On recherche l'impact d'une variation de tension d'entrée sur le système. Les références de vitesse et de courant d'axe d sont donc prises constantes (i.e. leurs variations sont supposées nulles) :

$$
\begin{aligned}
& {\left[\begin{array}{c}
\tilde{\mathrm{v}}_{\mathrm{d}}{ }^{*} \\
\tilde{\mathrm{v}}_{\mathrm{q}}{ }^{*}
\end{array}\right]=\left(\begin{array}{cc}
1 & \frac{-\omega_{0} \cdot \tau_{\mathrm{q}}}{1+\tau_{\mathrm{q}} \cdot \mathrm{s}} \\
\frac{\omega_{0} \cdot \tau_{\mathrm{d}}}{1+\tau_{\mathrm{d}} s} & 1
\end{array}\right) \text {. }} \\
& \left(-C_{q}(s) \cdot\left[\left(C_{v}(s) \cdot \frac{p \cdot \frac{\Psi_{f}}{f}}{1+T_{m} \cdot s}+1\right) \cdot \tilde{I}+C_{q}(s) \cdot k \cdot \frac{\frac{s}{\omega_{p}}}{\frac{s}{\omega_{p}}+1} \cdot \tilde{v} e\right]\right)
\end{aligned}
$$

où $\mathrm{f}$ est le coefficient de frottement visqueux, $\mathrm{J}$ l'inertie de l'ensemble tournant, $\mathrm{p}$ le nombre de paires de pôles, $\psi_{\mathrm{f}}$ le flux d'aimant et $\mathrm{T}_{\mathrm{m}}$ la constante de temps mécanique (J/f).

Les relations (2) et (3) conduisent alors à :

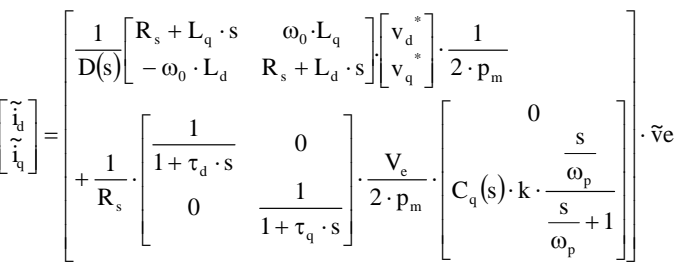

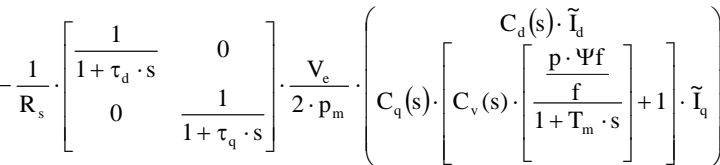

On note : $\tilde{\mathrm{i}}_{\mathrm{d}}=\mathrm{A}(\mathrm{s}) \cdot \hat{\mathrm{v}}_{\mathrm{e}}$ et $\tilde{\mathrm{i}}_{\mathrm{q}}=\mathrm{B}(\mathrm{s}) \cdot \hat{\mathrm{v}}_{\mathrm{e}}$ 
En combinant les relations (3) et (5) il vient alors :

$\frac{\tilde{\mathrm{v}}_{\mathrm{e}}}{\tilde{\mathrm{i}}_{\mathrm{e}}}=\mathrm{Z}_{\mathrm{e}}=-\frac{\mathrm{v}_{\mathrm{e}}}{\left(\mathrm{I}_{\mathrm{e}}-\mathrm{A}(\mathrm{s}) \cdot \mathrm{C}(\mathrm{s})-\mathrm{D}(\mathrm{s}) \cdot \mathrm{B}(\mathrm{s})\right)}$

\section{Calcul de l'impédance de sortie}

Pour modéliser la source d'énergie, on appelle L l'ensemble des inductances de fuite et/ou de câblage ramenées à l'entrée du pont redresseur, $\mathrm{R}$ la résistance modélisant l'ensemble des pertes résistives en série avec la source et $\mathrm{E}$ la tension du bus continu à vide (figure 6).

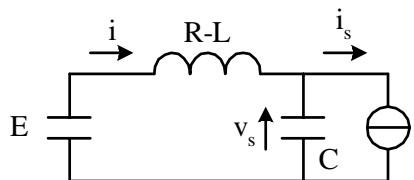

Figure 6. Modélisation de la source d’énergie

En l'absence de perturbations sur la tension d'entrée E, il vient :

$\mathrm{Z}_{\text {out }}=-\frac{\tilde{\mathrm{V}}_{\mathrm{s}}}{\tilde{\mathrm{i}}_{\mathrm{s}}}=\frac{(\mathrm{L} \cdot \mathrm{s}+\mathrm{R})}{\left(\mathrm{L} \cdot \mathrm{C} \cdot \mathrm{s}^{2}+\mathrm{R} \cdot \mathrm{C} \cdot \mathrm{s}+1\right)}$

D. Analyse de la stabilité de l'association filtre d'entrée - onduleur - machine synchrone

On fait l'hypothèse que tous les régulateurs sont de type PI. Les fonctions de transfert des correcteurs s'écrivent alors:

$$
\begin{aligned}
& \mathrm{C}_{\mathrm{d}}(\mathrm{s})=\frac{\mathrm{K}_{\mathrm{pd}} \cdot\left(1+\tau_{\mathrm{d}}{ }^{*} \cdot \mathrm{s}\right)}{\tau_{\mathrm{d}}{ }^{*} \cdot \mathrm{s}} \quad \mathrm{C}_{\mathrm{q}}(\mathrm{s})=\frac{\mathrm{K}_{\mathrm{pq}} \cdot\left(1+\tau_{\mathrm{q}}{ }^{*} \cdot \mathrm{s}\right)}{\tau_{\mathrm{q}}{ }^{*} \cdot \mathrm{s}} \\
& \mathrm{C}_{\mathrm{v}}(\mathrm{s})=\frac{\mathrm{K}_{\mathrm{v}} \cdot\left(1+\mathrm{T}_{\mathrm{m}}{ }^{*} \cdot \mathrm{s}\right)}{\mathrm{T}_{\mathrm{m}}{ }^{*} \cdot \mathrm{s}}
\end{aligned}
$$

Les paramètres du système concernent un ensemble filtre - onduleur machine synchrone à aimant connecté à un réseau de bord d'avion. La tension continue est issue du montage redresseur représenté sur la figure 3 . La machine synchrone est destinée à l'entraînement d'un actionneur.

Comme le prouve la figure 7 , avec les paramètres choisis, le système considéré est stable. Les paramètres de commande étant connus, l'inductance de fuite n'étant pas a priori facilement réglable, le seul paramètre permettant assurer la stabilité du système est la valeur de la capacité $C$. Pour que le système soit stable au point de fonctionnement désiré, celle-ci doit être supérieure à une valeur critique $\mathrm{C}_{\min }$. Sur la figure 8 est représentée l'évolution du maximum de la partie réelle des pôles de la fonction de transfert $\tilde{\mathrm{V}}_{\mathrm{s}} / \tilde{\mathrm{E}}$ en boucle fermée. On constate que le paramètre $\mathrm{K}$ a un effet légèrement stabilisant sur le système. La valeur de capacité pour laquelle, la mise en cascade de l'ensemble filtre d'entrée - onduleur - machine est stable, diminue lorsque $\mathrm{K}$ augmente mais ceci dans de faibles proportions. Sur la figure 9, on voit l'impact du choix de $\mathrm{K}$ sur l'ondulation de tension d'entrée lors d'une phase de démarrage. Les ondulations sont significativement diminuées.

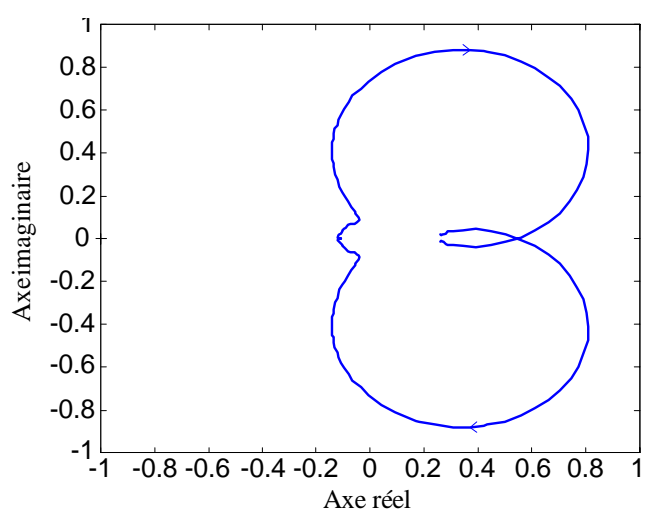

Figure 7. Tracé de Nyquist de $Z_{\text {out }} / Z_{\text {e }}$

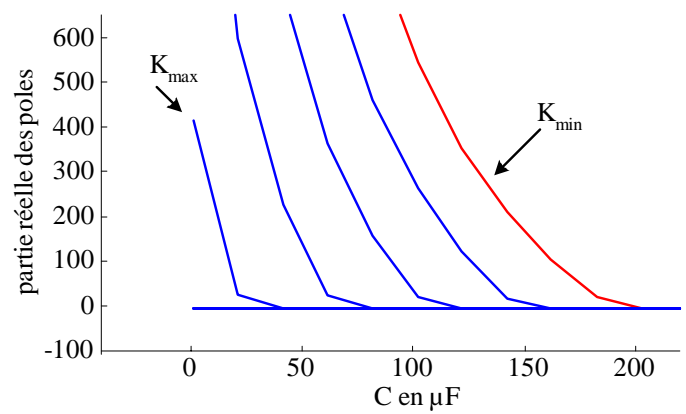

Figure 8 : Evolution du maximum des parties réelles des pôles lorsque $\mathrm{C}$ et $\mathrm{K}$ varient entre $\mathrm{K}_{\min }=0$ et $\mathrm{K}_{\max }=0,92$

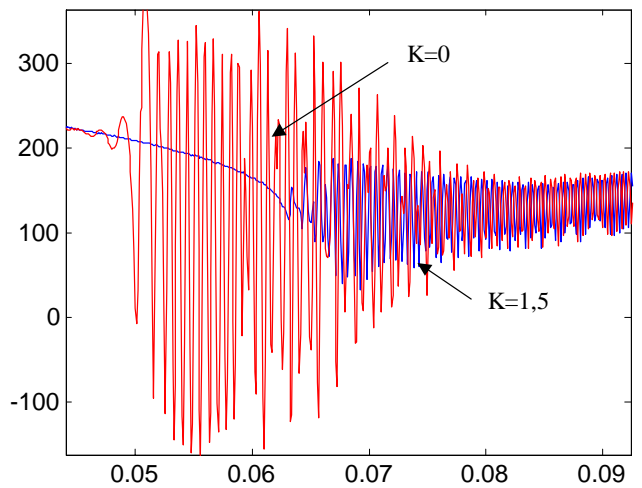

Figure 9 : Tension continue $\mathrm{v}_{\mathrm{s}}$ lors d'une phase de démarrage pour deux valeurs de $K$ ( $K=0$ en rouge et $K=1,5$ en bleue).

\section{E. Influence du mode de découplage}

Il est possible d'étudier l'impact du mode de découplage sur le dimensionnement de la capacité de stockage d'énergie C. Si l'on envisage maintenant un découplage de type Feedback. L'équivalent de la relation (2) conduit à :

$\left[\begin{array}{c}\tilde{\dot{i}_{d}} \\ \tilde{\mathrm{i}_{\mathrm{u}}}\end{array}\right]=\left[\begin{array}{cc}\frac{1}{\mathrm{R}_{\mathrm{s}}+\mathrm{L}_{\mathrm{d}} \cdot \mathrm{s}} & 0 \\ 0 & \frac{1}{\mathrm{R}_{\mathrm{s}}+\mathrm{L}_{\mathrm{q}} \cdot \mathrm{s}}\end{array}\right] \cdot\left(\frac{\tilde{\mathrm{v}}_{\mathrm{e}}}{2 \cdot \mathrm{p}_{\mathrm{m}}} \cdot\left[\begin{array}{c}\mathrm{v}_{\mathrm{d}}{ }^{*} \\ \mathrm{v}_{\mathrm{q}}{ }^{*}\end{array}\right]+\frac{\mathrm{V}_{\mathrm{e}}}{2 \cdot \mathrm{p}_{\mathrm{m}}} \cdot\left[\begin{array}{c}\tilde{\mathrm{v}}_{\mathrm{d}}{ }^{*} \\ \tilde{\mathrm{v}}_{\mathrm{q}}{ }^{*}\end{array}\right]\right)$ 
En suivant la méthodologie présentée en III.C, il est alors possible de calculer l'impédance d'entrée du dispositif avec ce type de couplage. La figure 10 représente l'évolution du maximum de la partie réelle des pôles du système en boucle fermée dans le cas de découplages Feedforward et Feedback avec $\mathrm{K}=0$. La valeur minimale de C assurant la stabilité du bus continu n'est que peu influencée par le mode de découplage choisi.

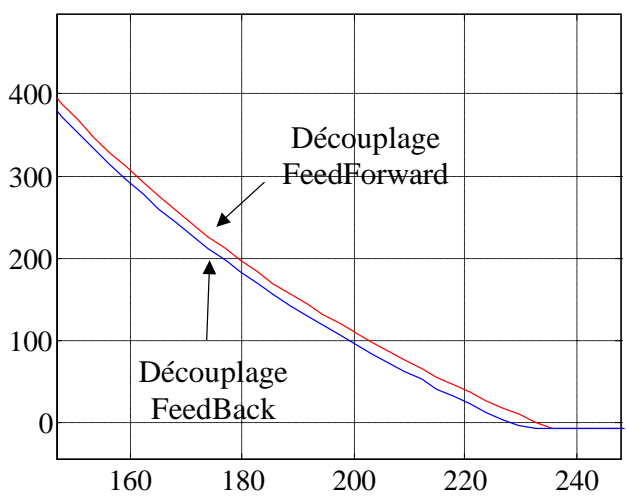

Figure 10. Evolution du maximum de la partie réelle des pôles en fonction du mode de découplage avec $\mathrm{K}=0$

IV. APPLICATION A UN ENSEMBLE REDRESSEUR SINUS CHARGE PAR N ENSEMBLES ONDULEUR - MACHINE SYNCHRONE A AIMANT

\section{A. Description générale de l'application}

Dans la partie précédente, le convertisseur de tête, un filtre d'entrée, était un dispositif passif. Il n'était donc pas "commandable". Lorsque le dispositif d'entrée est muni d'une commande, il est alors possible de rechercher des contrôles qui permettent d'obtenir une impédance de sortie quasi nulle dans la gamme de fréquence utile du système. Ceci permet alors de minimiser, voire d'éliminer tous les problèmes d'interaction et d'instabilité entre les divers convertisseurs mis en cascade et leurs charges. Dans cette partie, nous allons exposer une méthode d'usage général, qui permet d'exhiber une telle structure de contrôle. Les calculs sont réalisés dans le cas d'un convertisseur de tête de type redresseur commandé, chargé par $\mathrm{N}$ ensembles convertisseur - machine synchrone à aimant (figure 11). Une matrice de découplage est proposée rendant les commandes des charges indépendantes les unes des autres. Elle permet de plus d'assurer la stabilité du bus continu et ceci avec une faible valeur de capacité de stockage d'énergie.

\section{B. Mise en équation du système}

Les variations de tension du bus continu dépendent non seulement de la puissance injectée par le redresseur mais aussi des variations de charge dues aux moteurs. La structure de découplage doit assurer que les variations de tension continue $\widetilde{\mathrm{v}}_{\mathrm{S}}$ dépendent seulement des variations de la puissance injectée par le pont redresseur.

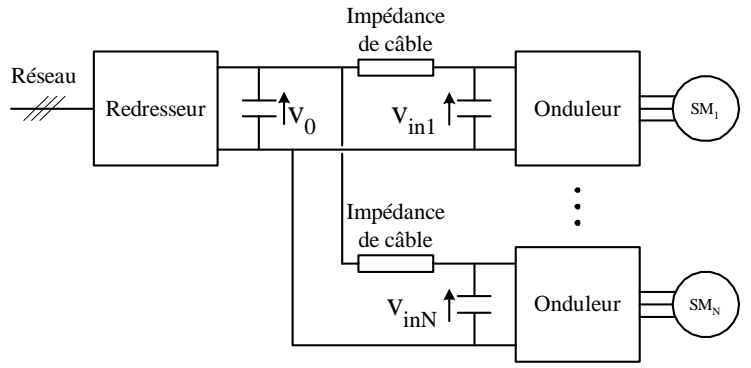

Figure 11. Schéma fonctionnel de l’application

De plus la variation de vitesse du $\mathrm{k}^{\text {éme }}$ moteur, notée $\tilde{\omega}_{\mathrm{k}}$, ne doit dépendre que de la variation du couple du $\mathrm{k}^{\text {éme }}$ moteur, lui-même propor-tionnel au courant d'axe q, $\tilde{\mathrm{i}}_{\mathrm{qk}}$ (cas des machines à pôles lisses). Ainsi la matrice de découplage doit permettre d'exprimer les variations des grandeurs de sortie sous la forme : $\tilde{\mathrm{v}}_{\mathrm{s}}=\mathrm{f}(\tilde{\mathrm{P}})$ et $\tilde{\omega}_{\mathrm{k}}=\mathrm{g}\left(\tilde{\mathrm{i}}_{\mathrm{qk}}\right)$.

Suite à une linéarisation au premier ordre des équations du système, la tension de sortie du pont redresseur vérifie la relation :

$$
\mathrm{C} \cdot \frac{\mathrm{d} \tilde{\mathrm{v}}_{\mathrm{s}}}{\mathrm{dt}}=\frac{\widetilde{\mathrm{P}}}{\mathrm{V}_{\mathrm{s}}}-\frac{\widetilde{\mathrm{P}}_{\text {cons }}}{\mathrm{V}_{\mathrm{s}}}
$$

où $\mathrm{C}$ est la capacité de stockage d'énergie et $\widetilde{\mathrm{P}}_{\text {cons }}$ la puissance totale fournie aux différentes charges :

$$
\widetilde{\mathrm{P}}_{\text {cons }}=\sum_{\mathrm{k}=1}^{\mathrm{N}} \widetilde{\mathrm{P}}_{\text {consk }}
$$

Si l'on fait l'hypothèse que la fréquence de commutation des interrupteurs est constante et que les pertes par effet Joule dans les câbles sont négligeables, il vient alors :

$\mathrm{P}_{\text {cons }}=\sum_{\mathrm{k}=1}^{\mathrm{N}}\left(\mathrm{V}_{\mathrm{dk}} \cdot \mathrm{I}_{\mathrm{dk}}+\mathrm{V}_{\mathrm{qk}} \cdot \mathrm{I}_{\mathrm{qk}}\right)$

En développant cette expression au premier ordre autour du point de fonctionnement du système, on a :

$$
\begin{aligned}
\tilde{\mathrm{P}}_{\text {cons }}= & \sum_{\mathrm{k}=1}^{\mathrm{N}} \mathrm{V}_{\mathrm{dk}} \cdot \tilde{\mathrm{i}}_{\mathrm{dk}}+\tilde{\mathrm{v}}_{\mathrm{dk}} \cdot \mathrm{I}_{\mathrm{dk}}+\mathrm{V}_{\mathrm{qk}} \cdot \tilde{\mathrm{i}}_{\mathrm{qk}}+\tilde{\mathrm{v}}_{\mathrm{qk}} \cdot \mathrm{I}_{\mathrm{qk}} \\
\text { avec: }: & \tilde{\mathrm{v}}_{\mathrm{dk}}=\left(\mathrm{R}_{\mathrm{sk}}+\mathrm{L}_{\mathrm{dk}} \cdot \mathrm{s}\right) \cdot \tilde{\mathrm{i}}_{\mathrm{dk}}-\omega_{\mathrm{ok}} \cdot \mathrm{L}_{\mathrm{qk}} \cdot \tilde{\mathrm{i}}_{\mathrm{qk}} \\
& \tilde{\mathrm{v}}_{\mathrm{qk}}=\left(\mathrm{R}_{\mathrm{sk}}+\mathrm{L}_{\mathrm{qk}} \cdot \mathrm{s}\right) \cdot \tilde{\mathrm{i}}_{\mathrm{qk}}+\omega_{\mathrm{ok}} \cdot \mathrm{L}_{\mathrm{dk}} \cdot \tilde{\mathrm{i}}_{\mathrm{dk}}
\end{aligned}
$$

où $\mathrm{L}_{\mathrm{dk}}=\mathrm{L}_{\mathrm{qk}}=\mathrm{L}_{\mathrm{k}}$ pour des moteurs lisses.

En supposant maintenant que la dynamique de l'asservissement en puissance est beaucoup plus rapide que celui en tension et en posant : $\widetilde{P}=G_{p} \cdot \widetilde{P}_{\text {ref }}$ (où $G_{p}$ représente le gain en puissance entre les variations de puissance de référence $\widetilde{\mathrm{P}}_{\text {ref }}$ et de puissance injectée $\widetilde{\mathrm{P}}$, on obtient : 


$$
\tilde{\mathrm{P}}_{\text {cons }}=\sum_{\mathrm{k}=1}^{\mathrm{N}}\left(\begin{array}{l}
{\left[\mathrm{V}_{\mathrm{dk}}+\left(\mathrm{R}_{\mathrm{sk}}+\mathrm{L}_{\mathrm{dk}} \cdot \mathrm{s}\right) \cdot \mathrm{I}_{\mathrm{dk}}+\omega_{\mathrm{ok}} \cdot \mathrm{L}_{\mathrm{dk}} \cdot \mathrm{I}_{\mathrm{qk}}\right] \cdot \tilde{\mathrm{i}}_{\mathrm{dk}}} \\
+\left[\mathrm{V}_{\mathrm{qk}}+\left(\mathrm{R}_{\mathrm{sk}}+\mathrm{L}_{\mathrm{qk}} \cdot \mathrm{s}\right) \cdot \mathrm{I}_{\mathrm{qk}}-\omega_{\mathrm{ok}} \cdot \mathrm{L}_{\mathrm{qk}} \cdot \mathrm{I}_{\mathrm{dk}}\right] \cdot \tilde{\mathrm{i}}_{\mathrm{qk}}
\end{array}\right)
$$

et :

$$
\tilde{\mathrm{v}}_{\mathrm{s}}=\frac{1}{\mathrm{~V}_{\mathrm{s}} \cdot \mathrm{C} \cdot \mathrm{s}} \cdot\left(\mathrm{G}_{\mathrm{p}} \cdot \tilde{\mathrm{P}}_{\mathrm{ref}}-\sum_{\mathrm{k}=1}^{\mathrm{N}}\left(\begin{array}{l}
\left.\left.\left[\mathrm{V}_{\mathrm{dk}}+\left(\mathrm{R}_{\mathrm{sk}}+\mathrm{L}_{\mathrm{dk}} \cdot \mathrm{s}\right) \cdot \mathrm{I}_{\mathrm{dk}}+\omega_{\mathrm{ok}} \cdot \mathrm{L}_{\mathrm{dk}} \cdot \mathrm{I}_{\mathrm{qk}}\right] \cdot \tilde{\mathrm{i}}_{\mathrm{dk}}+\mathrm{L}_{\mathrm{qk}} \cdot \mathrm{s}\right) \cdot \mathrm{I}_{\mathrm{qk}}-\omega_{\mathrm{ok}} \cdot \mathrm{L}_{\mathrm{qk}} \cdot \mathrm{I}_{\mathrm{dk}}\right] \cdot \tilde{\mathrm{i}}_{\mathrm{qk}}
\end{array}\right)\right.
$$

Les équations mécanique de la machine synchrone à aimant donnent alors :

$\mathrm{J}_{\mathrm{k}} \cdot \frac{\mathrm{d} \tilde{\omega}_{\mathrm{k}}}{\mathrm{dt}}=\mathrm{p}_{\mathrm{k}} \cdot \Psi_{\mathrm{fk}} \cdot \tilde{\mathrm{i}}_{\mathrm{qk}}-\mathrm{f}_{\mathrm{rk}} \cdot \tilde{\omega}_{\mathrm{k}}$

soit : $\tilde{\omega}_{\mathrm{k}}=\frac{\mathrm{p}_{\mathrm{k}} \cdot \Psi_{\mathrm{fk}}}{\left(\mathrm{f}_{\mathrm{rk}}+\mathrm{J}_{\mathrm{k}} \cdot \mathrm{s}\right)} \cdot \tilde{\mathrm{i}}_{\mathrm{qk}}$

où $\Psi_{\mathrm{fk}}$ est le flux magnétique à travers les enroulements statoriques, $\mathrm{p}_{\mathrm{k}}$ le nombre de paires de pôles du $\mathrm{k}^{\text {éme }}$ moteur, $\mathrm{J}_{\mathrm{k}}$ son inertie et où les variations du couple de charge sont supposée être proportionnelles à celles de vitesse. Sous forme matricielle, on obtient alors :

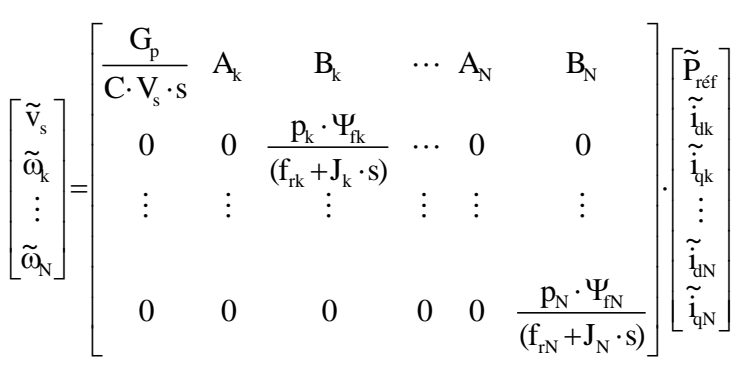

$$
\left[\begin{array}{c}
\widetilde{\mathrm{v}}_{\mathrm{s}} \\
\widetilde{\mathrm{i}}_{\mathrm{dk}} \\
\tilde{\mathrm{i}}_{\mathrm{qk}} \\
\vdots \\
\tilde{\mathrm{i}}_{\mathrm{dN}} \\
\tilde{\mathrm{i}}_{\mathrm{qN}}
\end{array}\right]=\left[\begin{array}{cccccc}
\frac{\mathrm{G}_{\mathrm{p}}}{\mathrm{C} \cdot \mathrm{V}_{\mathrm{s}} \cdot \mathrm{s}} & \mathrm{A}_{\mathrm{k}} & \mathrm{B}_{\mathrm{k}} & \cdots & \mathrm{A}_{\mathrm{N}} & \mathrm{B}_{\mathrm{N}} \\
0 & 1 & 0 & 0 & 0 & 0 \\
0 & 0 & 1 & 0 & 0 & 0 \\
0 & 0 & 0 & \ddots & 0 & 0 \\
0 & 0 & 0 & 0 & 1 & 0 \\
0 & 0 & 0 & 0 & 0 & 1
\end{array}\right] \cdot\left[\begin{array}{c}
\widetilde{\mathrm{P}}_{\mathrm{réf}} \\
\tilde{\mathrm{i}}_{\mathrm{dk}} \\
\widetilde{\mathrm{i}}_{\mathrm{qk}} \\
\vdots \\
\tilde{\mathrm{i}}_{\mathrm{dN}} \\
\tilde{\mathrm{i}}_{\mathrm{qN}}
\end{array}\right]
$$

Les termes $A_{k}$ et $B_{k}$ représentent les coefficients de couplage et sont égaux à :

$$
\begin{aligned}
& A_{k}=-\frac{\left[V_{d k}+\left(R_{s k}+L_{d k} \cdot s\right) \cdot I_{d k}+\omega_{o k} \cdot L_{d k} \cdot I_{q k}\right]}{V_{s} \cdot C \cdot s} \\
& B_{k}=-\frac{\left[V_{q k}+\left(R_{s k}+L_{q k} \cdot s\right) \cdot I_{q k}-\omega_{o k} \cdot L_{q k} \cdot I_{d k}\right]}{V_{s} \cdot C \cdot s}
\end{aligned}
$$

Dans cette relation, on appelle $\mathrm{P}_{\text {ref }}, \mathrm{V}_{\mathrm{dkref}}, \mathrm{V}_{\mathrm{qkref}}$ la puissance de référence et les tensions d'axes $\mathrm{d}$ et $\mathrm{q}$ de référence pour le $\mathrm{k}^{\text {éme }}$ moteur (à la sortie de la structure de découplage). De plus, on note $\mathrm{P}^{*}, \mathrm{~V}^{\prime}{ }_{\mathrm{dkref}}$, $\mathrm{V}^{\prime}{ }_{\text {qkref }}$ les sorties des boucles de tension et de courant d'axes d, q du k éme moteur.

On introduit une structure de découplage modélisée par une matrice $A$. Les équations de l'ensemble du système peuvent alors être mises sous la forme :

$$
\left[\begin{array}{c}
\tilde{\mathrm{P}}_{\mathrm{ref}} \\
\tilde{\mathrm{i}}_{\mathrm{dk}} \\
\tilde{\mathrm{i}}_{\mathrm{qk}} \\
\vdots \\
\tilde{\mathrm{i}}_{\mathrm{dN}} \\
\tilde{\mathrm{i}}_{\mathrm{qN}}
\end{array}\right]=[\mathrm{X}] \cdot[\mathrm{A}] \cdot[\mathrm{G}] \cdot\left[\begin{array}{c}
\tilde{\mathrm{P}}^{*} \\
\tilde{\mathrm{v}}_{\mathrm{dkref}}^{\prime} \\
\tilde{\mathrm{v}}_{\mathrm{qkref}}^{\prime} \\
\vdots \\
\tilde{\mathrm{v}}_{\mathrm{dNref}}^{\prime} \\
\tilde{\mathrm{v}}_{\mathrm{qNref}}^{\prime}
\end{array}\right] \begin{gathered}
\mathrm{A}=\left[\mathrm{a}_{\mathrm{l,c}}\right] \\
\text { où }: \mathrm{l} \in(1, \ldots, 1+2 \mathrm{~N}) \\
\mathrm{c} \in(1, \ldots, 1+2 \mathrm{~N})
\end{gathered}
$$

Les matrices $G$ et $X$ de dimension $(2 \mathrm{~N}+1) \times(2 N+1)$ représentent une matrice de gain et la matrice modélisant le système :

$[G]=\left[\begin{array}{cccccc}1 & 0 & 0 & 0 & 0 & 0 \\ 0 & G_{v} & 0 & 0 & 0 & 0 \\ 0 & 0 & G_{v} & 0 & 0 & 0 \\ 0 & 0 & 0 & \ddots & 0 & 0 \\ 0 & 0 & 0 & 0 & G_{v} & 0 \\ 0 & 0 & 0 & 0 & 0 & G_{v}\end{array}\right]$

$[\mathrm{X}]=\left[\begin{array}{cccccc}1 & 0 & 0 & 0 & 0 & 0 \\ 0 & \frac{\mathrm{R}_{\mathrm{s} 1}+\mathrm{L}_{\mathrm{q} 1} \cdot \mathrm{s}}{\mathrm{D}_{1}(\mathrm{~s})} & \frac{\omega_{\mathrm{o} 1} \cdot \mathrm{L}_{\mathrm{q} 1}}{\mathrm{D}_{1}(\mathrm{~s})} & 0 & 0 & 0 \\ 0 & \frac{-\omega_{\mathrm{o} 1} \cdot \mathrm{L}_{\mathrm{d} 1}}{\mathrm{D}_{1}(\mathrm{~s})} & \frac{\mathrm{R}_{\mathrm{s} 1}+\mathrm{L}_{\mathrm{d} 1} \cdot \mathrm{s}}{\mathrm{D}_{1}(\mathrm{~s})} & 0 & 0 & 0 \\ 0 & 0 & 0 & \ddots & 0 & 0 \\ 0 & 0 & 0 & 0 & \frac{\mathrm{R}_{\mathrm{sk}}+\mathrm{L}_{\mathrm{qk}} \cdot \mathrm{s}}{\mathrm{D}_{\mathrm{N}}(\mathrm{s})} & \frac{\omega_{\mathrm{ok}} \cdot \mathrm{L}_{\mathrm{qk}}}{\mathrm{D}_{\mathrm{N}}(\mathrm{s})} \\ 0 & 0 & 0 & 0 & \frac{-\omega_{\mathrm{ok}} \cdot \mathrm{L}_{\mathrm{dk}}}{\mathrm{D}_{\mathrm{N}}(\mathrm{s})} & \frac{\mathrm{R}_{\mathrm{sk}}+\mathrm{L}_{\mathrm{dk}} \cdot \mathrm{s}}{\mathrm{D}_{\mathrm{N}}(\mathrm{s})}\end{array}\right]$

où $G_{v}$ est le gain en tension de l'onduleur et :

$\mathrm{D}_{\mathrm{k}}(\mathrm{s})=\left(\mathrm{R}_{\mathrm{sk}}+\mathrm{L}_{\mathrm{qk}}\right) \cdot\left(\mathrm{R}_{\mathrm{sk}}+\mathrm{L}_{\mathrm{dk}}\right)+\omega^{2}{ }_{\mathrm{ok}} \cdot \mathrm{L}_{\mathrm{dk}} \cdot \mathrm{L}_{\mathrm{qk}}$

Pour assurer un contrôle découplé des courants d'axes d et q, une condition suffisante sur les coefficients de la matrice A est donnée par :

$$
\begin{array}{lrl}
a_{2 k, j}=0=a_{2 k+1, j} & \text { si }: \quad \begin{aligned}
j & \in\{1, \ldots, 2 N+1\} \\
j & \neq 2 k \quad j \neq 2 k+1
\end{aligned} \\
a_{2 k, 2 k}=1 & a_{2 k, 2 k+1}=-\frac{\omega_{o k} \cdot L_{d k}}{\left(R_{s k}+L_{d k} \cdot s\right)} \\
a_{2 k+1,2 k+1}=1 & a_{2 k+1,2 k}=\frac{\omega_{o k} \cdot L_{q k}}{\left(R_{s k}+L_{q k} \cdot s\right)}
\end{array}
$$

Ces relations correspondent aux résultats classiques obtenus par un découplage de type FeedForward des courants des machines. Si ces conditions sont vérifiées, la matrice précédente X.A.G peut être mise sous la forme : 


$$
X \cdot A \cdot G=\left[\begin{array}{cccccc}
a_{1,1} & \mathrm{a}_{1,2} \cdot \mathrm{G} & \mathrm{a}_{1,3} \cdot \mathrm{G} & \cdots & \cdots & \mathrm{a}_{1,2 \mathrm{~N}+1} \cdot \mathrm{G} \\
0 & \frac{\mathrm{G}}{\mathrm{R}_{\mathrm{sk}}+\mathrm{L}_{\mathrm{lk}} \cdot \mathrm{s}} & 0 & 0 & 0 & 0 \\
0 & 0 & \frac{\mathrm{G}}{\mathrm{R}_{\mathrm{sk}}+\mathrm{L}_{\mathrm{Lk}} \cdot \mathrm{s}} & 0 & 0 & 0 \\
0 & 0 & 0 & \ddots & 0 & 0 \\
0 & 0 & 0 & 0 & \frac{\mathrm{G}}{\mathrm{R}_{\mathrm{sN}}+\mathrm{L}_{\mathrm{tNN}} \cdot \mathrm{s}} & 0 \\
0 & 0 & 0 & 0 & 0 & \frac{\mathrm{G}}{\mathrm{R}_{\mathrm{sN}}+\mathrm{L}_{\mathrm{qN}} \cdot \mathrm{s}}
\end{array}\right]
$$

Pour découpler le contrôle de la tension continue $\mathrm{V}_{\mathrm{s}}$ des contrôles de vitesse de tous les moteurs, les coefficients de la première ligne de la matrice $\mathrm{A}$ doivent vérifier les conditions suffisantes suivantes :

$$
\left\{\begin{array}{l}
G_{v} \cdot a_{1,2 k} \cdot \frac{1}{C \cdot V_{s} \cdot s}+\frac{G_{v}}{R_{s k}+L_{d k} \cdot s} \cdot A_{k}=0 \\
G_{v} \cdot a_{1,2 k+1} \cdot \frac{1}{C \cdot V_{s} \cdot s}+\frac{G_{v}}{R_{s k}+L_{q k} \cdot s} \cdot B_{k}=0
\end{array}\right.
$$

Il vient alors :

$$
\begin{aligned}
& a_{1,2 k}=\frac{v_{d k}+\left(R_{s k}+L_{d k} \cdot s\right) \cdot i_{d k}+\omega_{o k} \cdot L_{d k} \cdot i_{q k}}{R_{s k}+L_{d k} s} \\
& a_{1,2 k+1}=\frac{v_{q k}+\left(R_{s k}+L_{q k} \cdot s\right) \cdot i_{q k}-\omega_{o k} \cdot L_{q k} \cdot i_{d k}}{R_{s k}+L_{q k} \cdot s}
\end{aligned}
$$

Il est alors possible de dimensionner les boucles de courants d'axes $d$ et $q$ pour chaque moteur. Leurs fonctions de transfert en boucle ouverte s'écrivent :

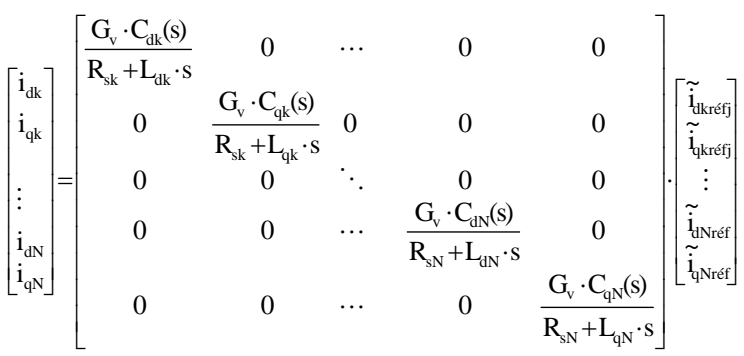

Si l'on suppose que les dynamiques des courants d'axes d, q sont beaucoup plus rapides que celles de la vitesse et de la tension continue, alors $\mathrm{i}_{\mathrm{dk}} \approx \mathrm{i}_{\mathrm{dkref}}$ et $\mathrm{i}_{\mathrm{qk}} \approx \mathrm{i}_{\text {qkref }}$. D'où $\widetilde{\mathrm{P}} \approx \mathrm{G}_{\mathrm{p}} \cdot \widetilde{\mathrm{P}}_{\text {ref }}$, il est alors possible de dimensionner séparément chaque variable lente grâce à la relation suivante :

$$
\left[\begin{array}{c}
\tilde{\mathrm{v}}_{\mathrm{s}} \\
\tilde{\omega}_{\mathrm{k}} \\
\vdots \\
\vdots \\
\vdots \\
\tilde{\omega}_{\mathrm{N}}
\end{array}\right]=\left[\begin{array}{cccc}
\frac{\mathrm{a}_{11} \cdot \mathrm{G}_{\mathrm{p}} \cdot \mathrm{C}_{\mathrm{v}}(\mathrm{s})}{\mathrm{C} \cdot \mathrm{V}_{\mathrm{s}} \cdot \mathrm{s}} & 0 & 0 & 0 \\
0 & \frac{\mathrm{p}_{\mathrm{k}} \cdot \Psi_{\mathrm{fk}}}{\left(\mathrm{f}_{\mathrm{rk}}+\mathrm{J}_{\mathrm{k}} \cdot \mathrm{s}\right)} & 0 & 0 \\
0 & 0 & \ddots & 0 \\
0 & 0 & 0 & \frac{\mathrm{p}_{\mathrm{N}} \cdot \Psi_{\mathrm{fN}}}{\left(\mathrm{f}_{\mathrm{rN}}+\mathrm{J}_{\mathrm{N}} \cdot \mathrm{s}\right)}
\end{array}\right] \cdot\left[\begin{array}{c}
\tilde{\mathrm{v}}_{\text {sref }} \\
\tilde{\mathrm{i}}_{\mathrm{qkréfj}} \\
\vdots \\
\vdots \\
\vdots \\
\tilde{\mathrm{i}}_{\mathrm{qNréf}}
\end{array}\right]
$$

\section{Résultats de simulation}

Pour réaliser la structure de découplage, des informations additionnelles représentées par les coefficients $\mathrm{a}_{1,2}, \ldots, \mathrm{a}_{1,2 \mathrm{~N}+1}$ doivent être envoyées à la commande du redresseur sinus. Les commandes de chaque moteur sont, elles, inchangées.

On se propose de vérifier les calculs précédents par simulation dans le cas de deux ensembles convertisseur - machine synchrone connectés à un même redresseur sinus. La commande de l'ensemble doit permettre la stabilisation du bus continu ainsi que la régulation de vitesse de chaque moteur. Les références de tension et de vitesse sont respectivement de $600 \mathrm{~V}$ et de $1500 \mathrm{tr} / \mathrm{min}$ et la capacité du condensateur à la sortie du redresseur est de $10 \mu \mathrm{F}$.

Les figures 12 et 13 soulignent l'effet du système de découplage proposé. Lors de variations de charge sur les moteurs, les variations de tension induites sur le bus continu sont négligeables. En fait, la tension de sortie du redresseur ne varie quasiment pas lors de perturbations provoquées par les deux convertisseurs de charge.
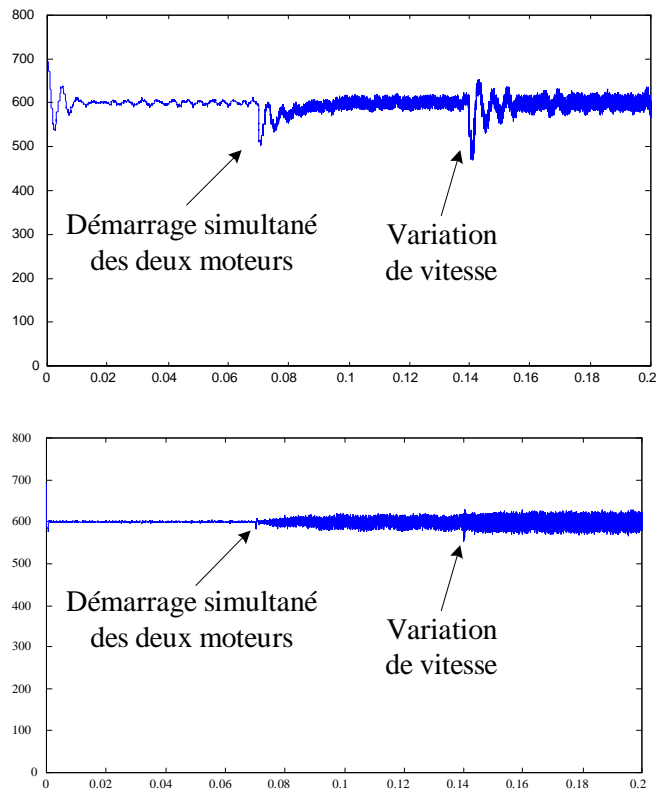

Figure 12. Tension du bus continu sans structure de découplage (en haut) et avec structure de découplage (en bas) 

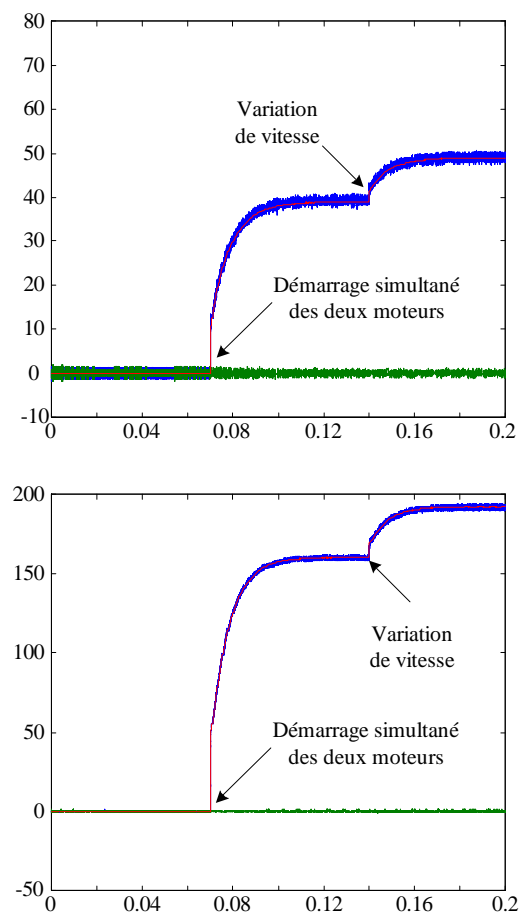

Figure 13. Courants d'axes $d$ et q pour les deux machines

L'impédance de sortie, vue par les deux charges est quasi nulle. Il est alors possible de diminuer sensiblement la taille de la capacité de stockage d'énergie $\mathrm{C}$ tout en conservant la stabilité du système.

De plus, on s'aperçoit, en regardant la structure de contrôle que le découplage de la tension du bus continu vis-à-vis des variations de vitesse ne dépend pas de la structure de contrôle de courant utilisée pour la commande des moteurs. Aucune information issue du redresseur n'est nécessaire pour le contrôle des deux moteurs. On en déduit alors que l'approche proposée reste valide quelle que soit la commande utilisée pour le contrôle du courant dans les moteurs.

Des essais réalisés en conservant la structure de découplage précédente pour la régulation de l'étage continu mais en remplaçant les contrôles des courants machines par des régulateurs non linéaires de type glissant ont montré que les propriétés de découplage sont conservées.

\section{CONCLUSION}

Le travail présenté ici traite des méthodes permettant l'étude de la stabilité des systèmes électriques interconnectés. Des critères d'impédance permettent, connaissant les caractéristiques de chaque dispositif, de prouver la stabilité du bus commun d'alimentation.
Un exemple traitant le cas d'un onduleur - machine synchrone à aimant muni de son filtre d'entrée a été présenté. On a montré que la dynamique des régulateurs de courant dans la machine a un impact fort sur la stabilité du bus continu. Le mode de découplage utilisé pour le contrôle des courants n'a lui, qu'un impact limité sur le dimensionnement du filtre d'entrée.

Cependant cette méthode établit des contraintes sur les dispositifs à connecter sur le bus commun d'alimentation (via leur impédance d'entrée). Lorsque tous les dispositifs sont munis d'une commande, il est possible d'annuler l'impédance de sortie de la source d'énergie pour chaque dispositif de charge. Un exemple traitant le cas d'un redresseur commandable à absorption sinusoïdale chargé par $\mathrm{N}$ ensembles convertisseur-machine synchrone a été présenté. L'approche proposée permet de prouver la stabilité du bus continu et de diminuer fortement la taille de ses capacités de stockage d'énergie. De plus la structure de découplage obtenue est d'usage général et peut être ajoutée à une structure de commande préexistante sans modification des commandes de tous les convertisseurs de charge.

\section{REFERENCES}

[1] C. M. Wildrick et F. C. Lee

A method of defining the load impedance specification for a stable distributed power system.

EEE Trans. on Power Electronics, vol. 10, $\mathrm{n}^{\circ} 3$, mai 1995, pp. 280-284.

[2] S. D. Sudhoff, D. H. Schmucker, R. A. Youngs et H. J. Hegner

Stability analysis of DC distribution systems using admittance space constraints.

Proceedings of The Institute of Marine Engineers All Electric Ship 98, London (UK), septembre 29-30, 1998.

[3] X. Feng et F. C. Lee

On-line measurement on stability margin of DC distributed power system.

APEC'00, Applied Power Electronics Conference and Exposition, New Orleans (USA), février 2000, vol. 2, pp. 1190-1196.

[4] X. Feng, C. Liu, Z. Ye , F. C. Lee et D. Borojevic Monitoring the stability of DC distributed power systems.

IECON'99, Industrial Electronics Conference, 1999, vol. 1, pp. 367-372.

[5] X. Feng, Z. Ye, K. Xing, F. C. Lee et D. Borojevic Impedance specification and impedance improvement for DC distributed power system.

PESC'99, Power Electronic Specialists Conference, Charleston (USA), juin 1999, vol. 2, pp. 889-894.

[6] X. Feng, Z. Ye, K. Xing, F. C. Lee et D. Borojevic Individual Load Impedance Specification for a Stable DC distributed power system.

APEC'99, Applied Power Electronics Conference and Exposition, Dallas (USA), mars 1999, vol. 2, pp. 923929. 